Pacific

Journal of

Mathematics

FLOWLINES TRANSVERSE TO KNOT AND LINK FIBRATIONS

RoBert GHRIST AND EIKo KIN

Volume $217 \quad$ No. 1

November 2004 


\title{
FLOWLINES TRANSVERSE TO KNOT AND LINK FIBRATIONS
}

\author{
Robert Ghrist And Eiko Kin
}

\begin{abstract}
A knot or link in $S^{3}$ is fibred if the complement fibres over $S^{1}$, the fibres being spanning surfaces. We focus on those fibred knots and links which have the following property: every vector field transverse to the fibres possesses closed flow lines of all possible knot and link types in $S^{3}$. Our main result is that a large class of fibred knots and links has this property, including all fibred nontorus 2-bridge knots. In general, sufficient conditions include a pseudo-Anosov type monodromy map and a sufficiently high degree of symmetry.
\end{abstract}

\section{Introduction}

We consider fibrations of knot or link complements in $S^{3}$ from the point of view of transverse vector fields.

Periodic flowlines of a vector field on $S^{3}$ possess knotting and linking information intimately related to the dynamical properties of the flow. Examples abound of classes of systems for which simple dynamics implicate simple knotted orbits (those whose complement cannot possess a hyperbolic structure) and vice versa: see [21] (Morse-Smale flows), [10] (Smale flows), $[\mathbf{7}, \mathbf{9}]$ (integrable Hamiltonian flows), [4] (the Lorenz system), and [8] (flows tangent to plane fields). In this paper, we demonstrate that complicated fibrations typically force complicated knot and link types in any transverse vector field - indeed, as complicated as can be imagined.

Consider a fibred knot or link $K$ in $S^{3}$; "fibred" means there is a fibration $\pi: S^{3}-K \rightarrow S^{1}$ where each fibre is a spanning surface $\Sigma$ for $K$. Choose any vector field $X$ transverse to the fibres of $\pi$ (in particular, $X$ must be nonvanishing). Birman and Williams originally asked the question, "Which knot types are forced to exist as periodic orbits of $X$ ?" The question splits into cases based on Thurston's classification theorem for surface maps applied to the monodromy $\Phi: \Sigma \rightarrow \Sigma$ of $\pi$. If the complement of $K$ is geometrically "simple" (e.g., the unknot, torus knots, iterated torus knots), then only a finite set of knot types are forced to exist for all $X$ (again, typically unknots, torus knots, and iterations). On the other hand, if the complement of $K$ is "hyperbolic" (in the sense that the monodromy map is of pseudo-Anosov 
type), there is always an infinite number of distinct knot types as periodic orbits in any vector field $X$ transverse to $\pi$.

The paper [5] carefully constructed a template (a branched surface with semiflow) in the complement of the figure-eight knot that captures all knot and link types forced by a transverse vector field. It was noted there that, although many knot types were present, others were seemingly impossible to locate. To the contrary, the paper [14] showed that this template is universal - every knot and link type can be found on this branched surface. It follows that any vector field transverse to a fibration of the figure-eight knot complement must possess all knot and link types as orbits. In this paper, we explore to what extent this property holds for other fibred knots and links.

Definition 1.1. A fibred knot/link $K \subset S^{3}$ is said to be universally fibred if each vector field $X$ transverse to the fibration possesses closed orbits of all knot and link types.

An obvious prerequisite for being universally fibred is that the monodromy be sufficiently complex: we must be in the pseudo-Anosov case. For in the case of periodic type, the forced links may have only a finite number of knot types represented (by suspending the periodic monodromy).

Our results are phrased in the language of braids: see $\S 2$ for details and Figure 1 for examples of braids. We summarize our results in a simplified form; see $\S 5$ for stronger results.

Main Theorem. Let $b$ be a braid of the form

$$
b=\sigma_{1}^{\epsilon_{1}} \sigma_{2}^{\epsilon_{2}} \cdots \sigma_{N}^{\epsilon_{N}},
$$

where $\epsilon_{i}=+1$ or -1 for each $i$ (see Figure 1, right) and not all the $\epsilon_{i}$ are the same. For every $m>1$, the closure of $b^{m}$ is a fibred knot/link in $S^{3}$ of pseudo-Anosov type. Furthermore:

(a) The closure of $b^{m}$ has the universal fibration property for all but finitely many $m$.

(b) The closure of $b^{2}$ always has the universal fibration property.

(c) Whenever $b$ contains the subword $\sigma_{i-1}^{-1} \sigma_{i} \sigma_{i+1}^{-1} \sigma_{i+2}\left(\right.$ or $\left.\sigma_{i-2} \sigma_{i-1}^{-1} \sigma_{i} \sigma_{i+1}^{-1}\right)$, the closure of $b^{m}$ is universally fibred for all $m>1$.

From result (b) above, we obtain a complete classification in the case of 2-bridge knots:

Corollary. Every fibred nontorus two-bridge knot has the universal fibration property.

The proof of the Main Theorem uses a construction initiated in [5], based on work of Goldsmith [15] (and, ultimately, on work of Alexander). This 

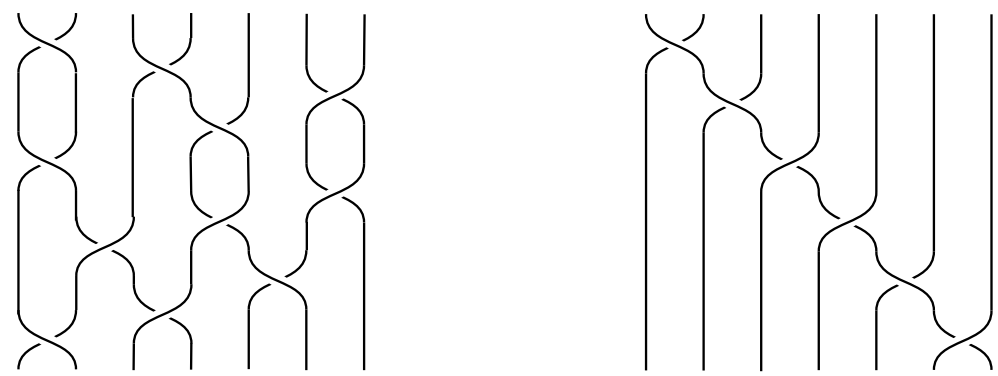

Figure 1. Left: an example of a braid on seven strands. Right: an example of a braid represented by Equation (1), with $b=\sigma_{1} \sigma_{2} \sigma_{3}^{-1} \sigma_{4}^{-1} \sigma_{5} \sigma_{6}^{-1}$.

involves taking a branched cover of $S^{3}$ over an unknot and lifting a branched surface constructed from a train track.

The outline of the paper is as follows: in $\S 2$, we review basic definitions and tools needed for the proofs. In $\S 3$, we introduce a family of pseudoAnosov braid types and present their train tracks. We proceed with the general template constructions in $\S 4$, and identify universal subtemplates to prove the Main Theorem in $\S 5$. The final section, $\S 6$, gives a summary of the extent to which we can classify the universally fibred knots and links. We propose a class of pseudo-Anosov fibrations that we believe do not induce all knot and link types in the transverse flow.

\section{Background}

Several of the topics below require entire books to do the material justice: we limit ourselves to the minimal set of ideas necessary to follow the remaining sections.

2.1. Fibred knots and links. A knot/link $K$ is said to be fibred if there is a fibration $\pi: S^{3}-K \rightarrow S^{1}$ with fibre a spanning surface $\Sigma$ for $K$. The complement of a neighborhood of $K$ in $S^{3}$ is homeomorphic to the mapping torus of a homeomorphism $\Phi: \Sigma \rightarrow \Sigma$ : that is,

$$
S^{3}-N(K) \cong(\Sigma \times[0,1]) /(x, 0)=(\Phi(x), 1) .
$$

The spanning surface $\Sigma$ is called the fibre surface, and the homeomorphism $\Phi$, which is well-defined up to isotopy, is called the monodromy. The union of the closed orbits of the suspension flow by $\Phi$ on $S^{3}-N(K)$ is called a planetary link of $\Phi$.

It follows from the Thurston classification theorem for surface homeomorphisms [20] that the monodromy of a fibred knot/link is isotopic to $\Phi^{\prime}$, a map of one of three types: 
1) periodic: $\left(\Phi^{\prime}\right)^{n}=$ ID for some $n$;

2) pseudo-Anosov: $\Phi^{\prime}$ possesses a pair of transverse (singular) foliations $\lambda^{u}$ and $\lambda^{s}$ along which $\Phi^{\prime}$ is uniformly expanding and contracting respectively;

3) reducible: there is a collection of disjoint simple closed curves $\mathcal{C} \subset \Sigma$ such that $\Phi^{\prime}(\mathcal{C})=\mathcal{C}$, and each connected component of $\Sigma-\mathcal{C}$ has negative Euler characteristic.

We say that a fibred knot/link $K$ is of periodic, pseudo-Anosov, or reducible type if its monodromy map is isotopic to a map of the corresponding type.

We will be most interested in those fibred knots/links of pseudo-Anosov type, such as the figure-eight knot, since these alone may permit universal fibrations. We can reduce the problem to the case where the monodromy is the pseudo-Anosov representative thanks to a theorem of Asimov and Franks [1] that guarantees that any map isotopic to a pseudo-Anosov map (and hence any vector field transverse to the fibration) cannot remove any of these periodic orbits - isotoping the monodromy can generate more, but not less. Hence, the set of planetary links of the pseudo-Anosov map is "minimal" with regard to changing the monodromy map (or the vector field). Henceforth, by the planetary link of a knot/link we mean the planetary link of its pseudo-Anosov monodromy map.

2.2. Braids and branched covers. We use the language of braids for the remainder of the paper. Recall that braids are isotopy classes of disjointly embedded arcs monotonically connecting fixed endpoints (as in Figure 1). Braids on $n$ strands form a group $B_{n}$ under concatenation, with standard generators $\sigma_{i}$ denoting an elementary crossing of the $i$ - th over the $(i+1)$-st strand. Inverses correspond to reversing the crossing of the sign. ${ }^{1} \mathrm{~A}$ closed or geometric braid is obtained by joining the two sets of endpoints around some fixed braid axis: algebraically, this corresponds to taking the conjugacy class of the braid in the braid group.

The classification of monodromies into periodic, pseudo-Anosov, or reducible type has its analogue for braids. Let $\beta$ denote the closure of $b$, an $n$-braid with braid axis $\alpha$. Since $\alpha$ is unknotted, its complement fibres over $S^{1}$ with fibre a disc $A$ meeting $\beta$ at $n$ points. The monodromy map on $A$ is fixed on the boundary and, by removing the intersection with $\beta$, becomes a map on an $n$-punctured disc. The Thurston Classification theorem applied to this monodromy map implies that one may classify a braid $b$ as being of periodic type, pseudo-Anosov type, or reducible type. Pseudo-Anosov type braids will be of central importance in analyzing fibrations.

While fibrations of knot complements are difficult to visualize (the explanations of the trefoil in [19, Section 10I] and of the figure-eight knot in [5]

\footnotetext{
${ }^{1}$ There is a tradition of dynamicists and topologists using opposite sign conventions for braids: we employ the dynamicists' convention and apologize for the inevitable annoyance.
} 
are not short!), the branched covering construction of Goldsmith [15] and Birman [3] provides a concrete mechanism for analysis. We use the method as presented in [5, pp. 26-30], keeping similar notation.

Lemma 2.1 ([15, Lemma 1]). Let $\beta$ be a single-component unknotted geometric braid with braid axis $\alpha$ and meridional disc $A$ spanning $\alpha$. Let $p: S^{3} \rightarrow S^{3}$ be a branched covering space projection whose branch set is $\beta$. Then $\underline{\alpha}=p^{-1}(\alpha)$ is a nontrivial fibred knot/link in $S^{3}$ with fibre $\underline{A}=p^{-1}(A)$.

A result of Birman [3] shows that every fibred knot may be obtained as $p^{-1}(\alpha)$ for some $\beta$ and some (irregular) cover $p$. Moreover, the fibration of the complement of $\alpha$ is itself a lift of the fibration of the complement of $\alpha$.

For the remainder of this paper, we work with pairs of curves $(\alpha, \beta)$ that are exchangeable; this means there is an isotopy of $S^{3}$ that presents $\alpha$ as a geometric $n$-braid with braid axis $\beta$ and vice versa [17]. Let $a$ be the braid in $B_{n}$ whose closure is $\alpha$, with $\beta$ as its axis, and let $b$ be the braid whose closure is $\beta$ with axis $\alpha$. In this case, $\underline{\alpha}$ is the closure of $a^{m}$ if $p$ is $m$-fold.

2.3. Train tracks and templates. The problem of how to understand the complex dynamics associated to a pseudo-Anosov surface homeomorphism was resolved with the theory of train tracks. The central idea, going back to Williams [22], is to project the foliation down to a branched manifold. We call attention to $[\mathbf{1 8}, \mathbf{2}]$ for treatments of this theory from different perspectives.

Recall that a pseudo-Anosov surface map is characterized by the pair of transverse singular foliations, $\lambda^{s}$ and $\lambda^{u}$, along which the map is uniformly contracting and expanding respectively. Roughly speaking, a train track for a pseudo-Anosov map is a branched 1-manifold obtained by cutting open the singular foliation $\lambda^{s}$ in a certain manner and collapsing each leaf to a point: see $[\mathbf{1 2}]$ for examples relevant to this paper. The dynamics of the pseudo-Anosov homeomorphism is then easily represented by an induced self-immersion of the train track. We note that there are several presentations available for train tracks. For the remainder of this paper, we follow $[\mathbf{2}, \mathbf{6}]$, for example, and represent the train track as a graph. The induced map is then easily represented as a map taking each edge of the graph to a sequence of edges. Two simple examples of train track graphs and associated graph maps are illustrated in Figure 10.

The problem of how to capture all of the knot and link data of a dynamically complex 3-dimensional flow was successfully tackled by Birman and Williams in the early 1980s $[\mathbf{4}, \mathbf{5}]$ in the theory of templates (a.k.a. "knotholders").

Definition 2.2. A template is an embedded branched 2-manifold with boundary, outfitted with an expansive semiflow. Templates have a natural decomposition into a finite number of branchline charts (Figure 2, left), each 
containing a number (greater than 1) of incoming and outgoing strips whose ends are joined respecting the direction of the semiflow.

The term "semiflow" means that the flow is well-defined in forward time, but not in backward time (at the branch lines in particular - there is a loss of uniqueness). By "expansive," it is meant that the semiflow expands volume everywhere.
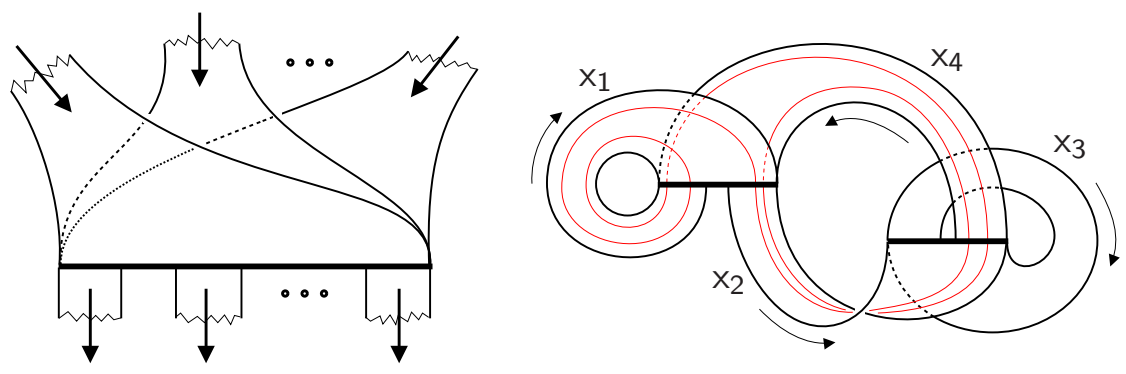

Figure 2. A branchline chart for a template (left); an example of a template with an unknotted periodic orbit on it (right). In the alphabet $\left\{\mathrm{x}_{1}, \mathrm{x}_{2}, \mathrm{x}_{3}, \mathrm{x}_{4}\right\}$, this knot corresponds to the word $\left(x_{1}^{2}\left(x_{2} x_{4}\right)^{2}\right)=\left(x_{1} x_{1} x_{2} x_{4} x_{2} x_{4}\right)$.

Every template carries an infinite link of periodic orbits of the semiflow. There is a well-defined symbolic language for describing these orbits. If each strip of a template $\mathcal{T}$ is given a label $x_{i}$, each forward orbit of the semiflow on $\mathcal{T}$ acquires an associated semiinfinite word in the alphabet $\left\{x_{i}\right\}$. From the expansivity of the semiflow, it can be shown that periodic flowlines on $\mathcal{T}$ are in fact in bijective correspondence with (admissible) periodic words in this alphabet, up to cyclic permutations of the words (see [5] or [13, Lemma 2.4.1] for details).

The Template Theorem [5, Theorem 2.1] of Birman and Williams gives very general conditions under which the dynamics of a three-dimensional flow is accurately captured by a template: see [13] for a comprehensive treatment. For our purposes, we note that for a fibred knot/link $K$ with pseudo-Anosov monodromy, there is a template $\mathcal{T} \subset S^{3}-K$ whose knot and link types are in bijective correspondence with those of the flow except for a finite number of orbits on the boundary of the template. The template is a faithful representation of the fibration: not only are knot/link data preserved, but also the periods of these orbits with respect to the monodromy map are captured as the intersection number of the orbits with the branch set of the template.

The template is obtained by suspending the train track graph map for the monodromy, and cutting along the vertices of the graph as necessary. These 
cuts may produce "extra" orbits on the templates not in the original flow. To minimize confusion, we note that in the remainder of this paper, any such exceptional orbits which we "lose" are unknots, and are easily found elsewhere in the interior of the template.

Definition 2.3. A universal template in $S^{3}$ is one that possesses all knot and link types as closed orbits of the semiflow.

The paper [14] established that universal templates exist in abundance. From that work and [13] comes a practical criterion for determining when a given template is universal. One additional definition is needed. Given any closed orbit $\kappa$ on a template $\mathcal{T} \subset S^{3}$, the twist, $\tau(\kappa)$, is defined to be the twist number of the normal bundle to $\mathcal{T}$ along $\kappa$ (which is either an annulus or Möbius band having $\kappa$ as its core). For the remainder of this paper, we consider twists for unknotted curves only, avoiding any ambiguity in how to count twist: an unknot with zero twist is one whose normal bundle can be isotoped to be a plane annulus.

The following criterion is a very slight modification of [13, Corollary 3.2.17], with a near-identical proof. Since the method of proof relies heavily on terminology and techniques of [13], we suppress the details.

Theorem 2.4. Let $\mathcal{T}$ be a template in $S^{3}$. Suppose that there exist three disjoint closed orbits $\kappa, \kappa^{\prime}, \kappa^{\prime \prime}$ on $\mathcal{T}$ such that:

1) They are separable unlinked unknots.

2) $\tau(\kappa)=0, \tau\left(\kappa^{\prime}\right)>0, \tau\left(\kappa^{\prime \prime}\right)<0$.

3) These three unknots intersect some branchline of $\mathcal{T}$ as in Figure 3, left, with the specified adjacencies and strip crossings.

Then $\mathcal{T}$ is a universal template.

The proofs of all the main theorems in this paper consist of constructing and simplifying templates for fibred knots and links, and then searching for the three unknots $\left\{\kappa, \kappa^{\prime}, \kappa^{\prime \prime}\right\}$ to which one may apply Theorem 2.4. Because these templates are complex, we introduce a "cartoon" notation [16]:

1) Strips in the template are collapsed along the direction transverse to the semiflow to become braided curves, except in a neighborhood of the branch lines.

2) Each half-twist of each strip is represented by a label + or - within a box, depending on the sign of the crossing.

3) Templates will often be "cut open" with the top and bottom to be identified.

For example, in Figure 3, the template shown in the center is expressed in cartoon form on the right.

2.4. Templates for planetary links. The branched cover construction allows one to construct a template for the planetary link of certain fibred 

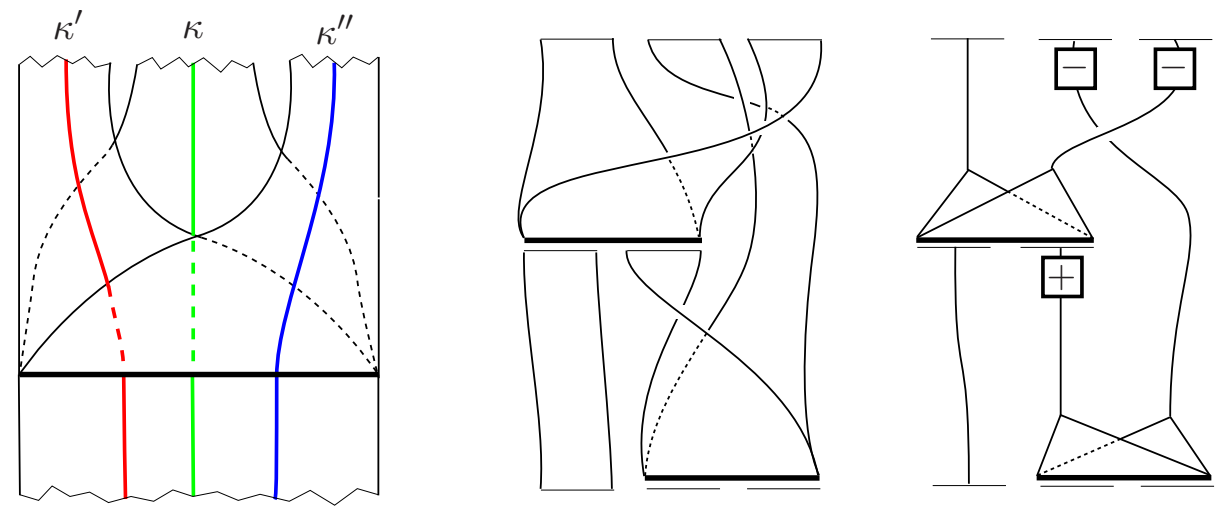

Figure 3. Left: $\kappa^{\prime}, \kappa, \kappa^{\prime \prime}$ from left to right. Center and right: a template and its cartoon representation; top and bottom are identified.

knots/links. Recall the notation introduced earlier: $\beta$, the closure of $b$, is an unknotted geometric braid with braid axis $\alpha ; p$ is an $m$-fold branched cover over $\beta$; and $\underline{\alpha}=p^{-1}(\alpha)$ is the lift of the braid axis, which, in the case of exchangeable braids is the closure of the braid $a^{m}$.

We summarize the procedure of [5, pp. 26-30] in the case of an exchangeable braid. If $b$ is a braid of pseudo-Anosov type, $\underline{\alpha}$ is fibred and also of pseudo-Anosov type, since the monodromy map for $\underline{\alpha}$ projects under $p$ to the monodromy map of $\alpha$ represented by the pseudo-Anosov braid type $b$.

The following procedure yields a template for the fibration of the closure of $a^{m}$ :

1) Construct a train track graph for $b$ along with its induced map.

2) Suspend the train track graph map and cut as necessary to obtain a template $\mathcal{T}$ in the solid torus $S^{3}-\alpha$.

3) Peel off a copy of the unknot $\beta$ from the boundary of $\mathcal{T}$ and find a spanning disc $D$ for this unknot.

4) Cut $\mathcal{T}$ along $D$ and glue $m$ copies of this cut template together endto-end cyclically to obtain $\mathcal{U}^{m}$, the template for the fibration of the complement of $\underline{\alpha}$.

This procedure was carried out carefully in [5, pp. 28-30] for the figure-eight knot, which is the closure of the braid $\left(\sigma_{1} \sigma_{2}^{-1}\right)^{2}$. In this case $a=b=\sigma_{1} \sigma_{2}^{-1}$ is the simplest pseudo-Anosov braid type. The train track graph for this braid is an interval, and the graph map is represented in Figure 10, left. We reproduce this example in cartoon notation in Figure 4 in preparation for the proof of part (c) of the Main Theorem. Familiarity with this example will reveal patterns in the general case. 

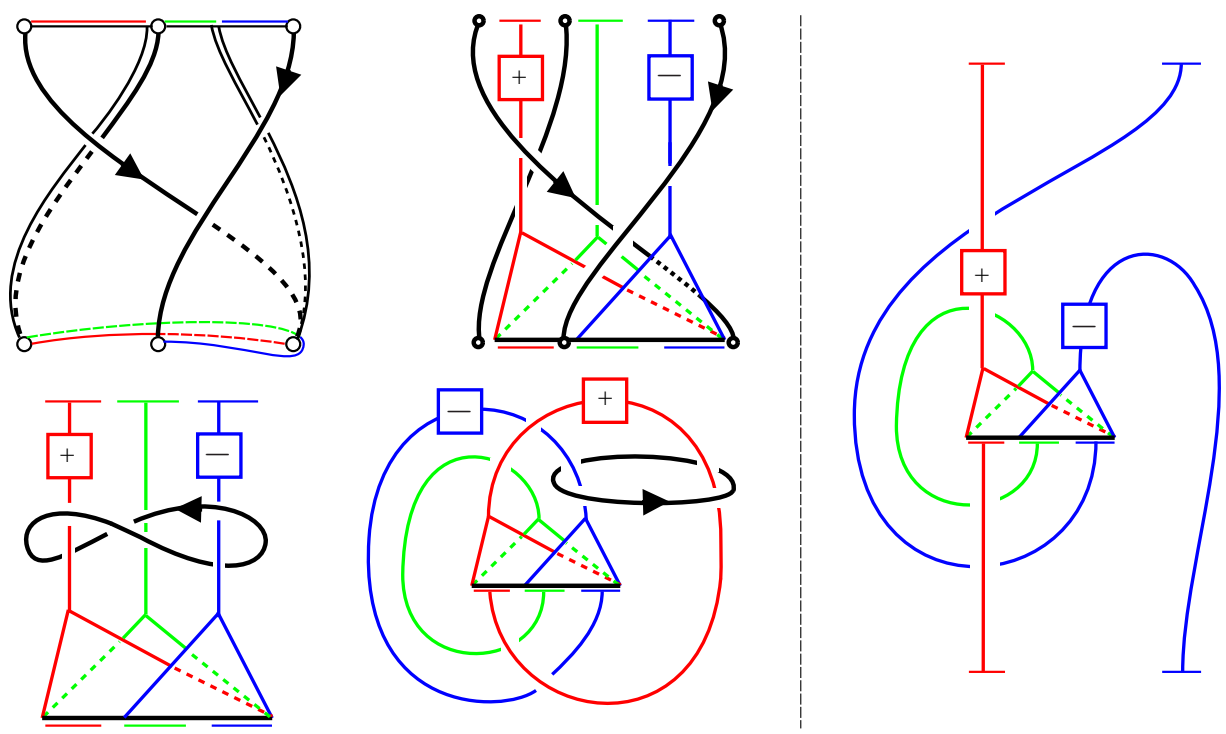

Figure 4. The branched cover construction for the figureeight knot complement. The suspension of the train track for the braid $b=\sigma_{1} \sigma_{2}^{-1}$ is a template (upper left). By peeling off a copy of $\beta$ and cutting along its spanning disc (lower left), one has a fundamental domain for the template of the $m$-fold cover (right).

\section{Families of fibred knots and links}

We focus on the following families of braids along which to perform the branched cover construction:

Definition 3.1. Given integers $n_{1}, n_{2}, \ldots, n_{k} \geq 1$ (with $k>1$ ), denote by $b:=b_{\left(n_{1}, \ldots, n_{k}\right)}$ the braid on $\left(1+\sum_{r=1}^{k} n_{r}\right)$ strands given by the braid word

$$
\underbrace{\sigma_{1} \sigma_{2} \cdots \sigma_{n_{1}}}_{n_{1}} \underbrace{\sigma_{n_{1}+1}^{-1} \cdots \sigma_{n_{1}+n_{2}}^{-1}}_{n_{2}} \cdots \underbrace{\sigma_{1+\sum_{r=1}^{(-1)} n_{r}^{(k+1)}}^{\sigma_{1-1}} \cdots \sigma_{\sum_{r=1}^{k} n_{r}}^{(-1)^{(k+1)}}}_{n_{k}} .
$$

Thus, $b$ is equivalent to the form given in (1). For example, the braid in Figure 1 is $b_{(2,2,1,1)}$. Clearly, one can also use the mirror image of these braids, and all the theorems we prove hold here as well.

Lemma 3.2. The closure of $b_{\left(n_{1}, \ldots, n_{k}\right)}$ is self-exchangeable. Specifically, given $\beta$ the closure of $b_{\left(n_{1}, \ldots, n_{k}\right)}$ with braid axis $\alpha$, there exists an isotopy of $S^{3}$ taking $\alpha$ to the closure of $b_{\left(n_{1}, \ldots, n_{k}\right)}$ with braid axis $\beta$. 
Proof. We induct on the length of the braid word $b$. Assume as an induction hypothesis that the $N$-strand closed braid $\beta$ exchanges with $\alpha$ as in Figure 5, left: specifically, that there is an isotopy fixed on a solid ball as indicated. Now, for a braid of the form $b \sigma_{N}$ (in Figure 5 , right) or $b \sigma_{N}^{-1}$, fit the final strand within a larger fixed solid ball and perform the isotopy given by induction. Figure 6 shows that this final strand of $\beta$ may be exchanged relative to a yet smaller fixed ball. (In the diagram, the set of crossings in the braid word $b$ is flipped and rotated, but is kept together rigidly.)
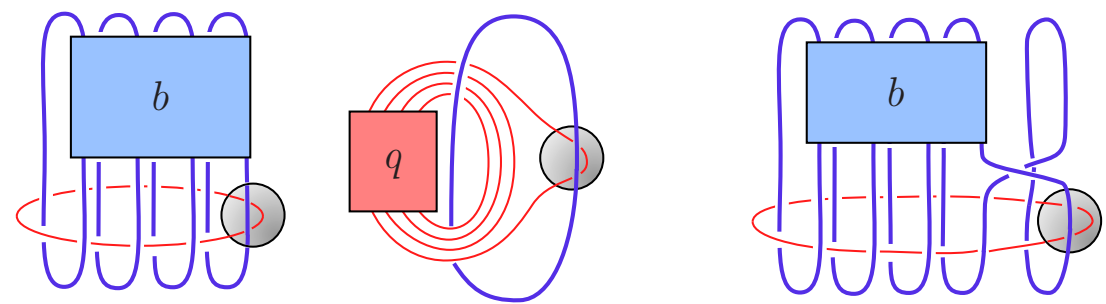

Figure 5. The induction hypothesis: the closure of the braid $b$ can be exchanged with its axis rel a fixed ball (left); the setup for the induction step (right).
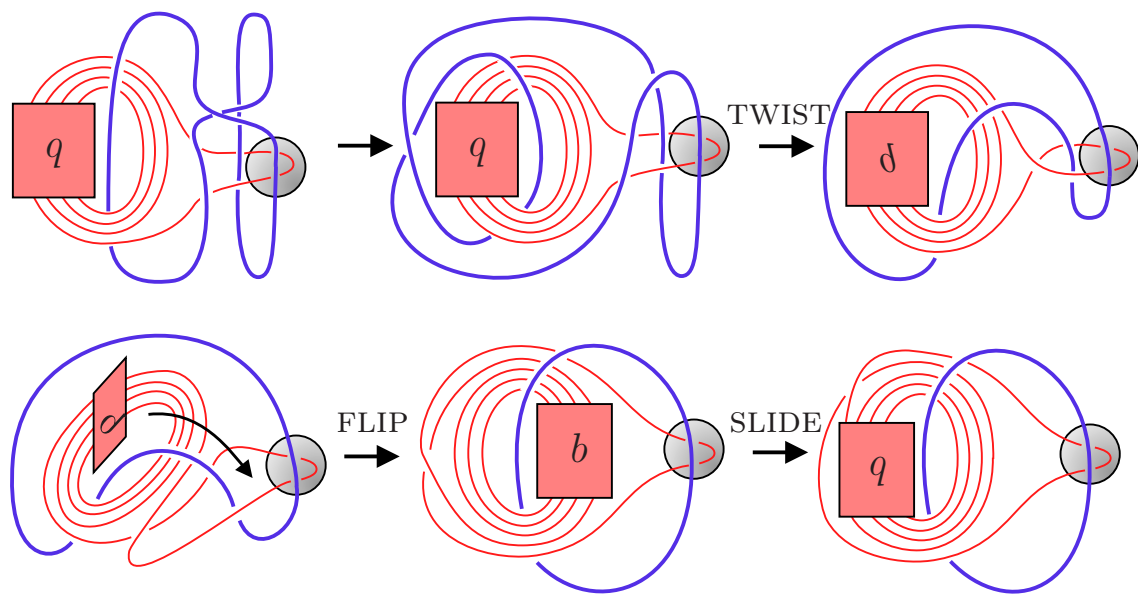

Figure 6. The induction step: the closure of $b \sigma_{N}$ can be exchanged with its axis rel a fixed ball.

Lemma 3.3. The braid $b=b_{\left(n_{1}, \ldots, n_{k}\right)}$ is of pseudo-Anosov type. 
A proof of this can be explicitly given by one of several algorithms available. $^{2}$ We note, however, that the braid $b$ is precisely that considered by Gabai and Kazez [12], who show the pseudo-Anosov braid type.

By Lemma 3.3, we have:

Corollary 3.4. For each integer $m>1$, the closure of $\left(b_{\left(n_{1}, \ldots, n_{k}\right)}\right)^{m}$ is a fibred knot/link of pseudo-Anosov type.

The braid $b_{\left(n_{1}, \ldots, n_{k}\right)}$ induces a very simple map on the closed disc, consisting of $k$ rotations in alternate directions, and the associated train track is straightforward to compute. Let $G=G_{\left(n_{1}, \ldots, n_{k}\right)}$ be a chain of $k$ radial "stars" $S_{1}, \ldots, S_{k}$, each having a vertex of valence $\left(n_{i}+1\right)$ if $n_{i}>1$ (see Figure 7 ). The radial stars alternate their up-down orientation, and small loops (corresponding to the periodic orbit) are attached at the ends of the radial oriented edges as in Figure 7 . The set of nonloop edges in $S_{i}$ equals $\left\{e_{i}^{0}\right\}$ if $n_{i}=1$, and $\left\{e_{i}^{0}, \ldots, e_{i}^{n_{i}}\right\}$ if $n_{i}>1$. For examples, see Figure 8 .
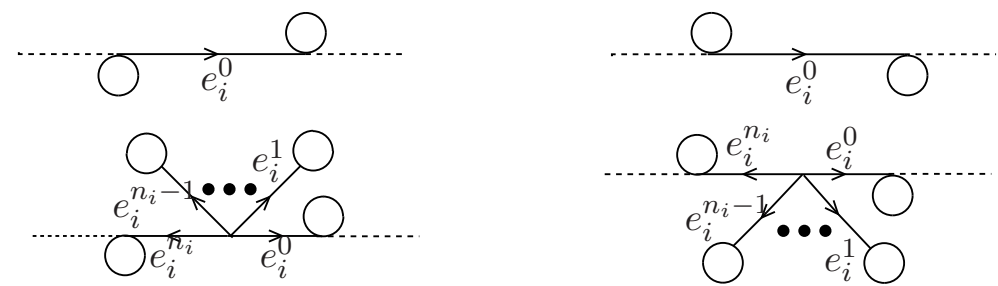

Figure 7. Depiction of $g$. Left column: $i$ odd; rigth column: $i$ even. Upper row: $n_{i}=1$; lower row: $n_{i}>1$.
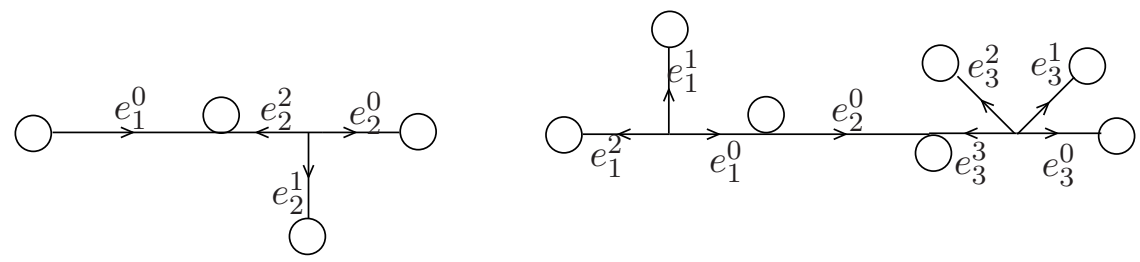

Figure 8. Left: $G_{(1,2)}$. Right: $G_{(2,1,3)}$.

The next statement uses the notion of efficient graph maps, for whose definition we refer to [2, p. 114]. In general, the efficient graph map is not unique for a braid.

\footnotetext{
${ }^{2}$ We have used the Bestvina-Handel algorithm [2] for dealing with this family: though straightforward, the algorithm is lengthy and of little consequence for the remainder of this article.
} 
Lemma 3.5. An efficient graph map $g=g_{\left(n_{1}, \ldots, n_{k}\right)}: G \rightarrow G$ for the train track graph of $b_{\left(n_{1}, \ldots, n_{k}\right)}$ is given as $g_{k} \circ \cdots \circ g_{1}$, where $g_{i}$ rotates the $i$-th star $S_{i}$ clockwise if $i$ is even and counterclockwise if $i$ is odd. (See Figure 9.)
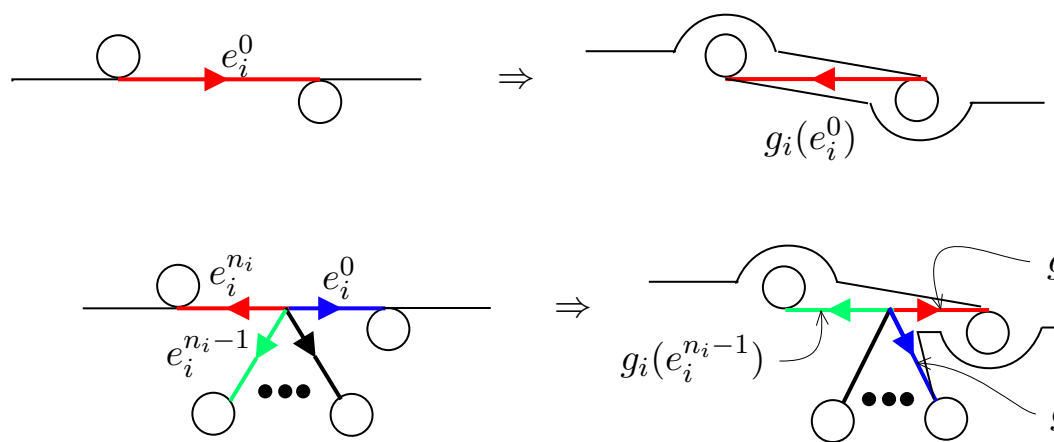

Figure 9. Action of $g$. Top: $i$ even, $n_{i}=1$. Bottom: $i$ even, $n_{i}>1$.

The algebraic presentation of the train track map is simple to derive knowing that it is composed of rotations. For simplicity, we ignore all loopedges in the train track. Given an oriented edge $E$, we denote the same edge oriented in the opposite direction by $\bar{E}$. The following lemma is used to derive a normal form of the template $\mathcal{T}_{(p, q)}$ in Section 4.1.

Lemma 3.6. The images of the edges under $g=g_{(p, q)}$ are as follows: For $p=q=1$,

$$
\begin{aligned}
& g\left(e_{1}\right)=\overline{e_{2}^{0}} \overline{e_{1}^{0}}, \\
& g\left(e_{2}\right)=e_{1}^{0} e_{2}^{0} \overline{e_{2}^{0}} .
\end{aligned}
$$

For $p, q>1$,

$$
\begin{aligned}
& g\left(e_{1}^{p}\right)=e_{1}^{0} \overline{e_{2}^{q}} e_{2}^{0}, \\
& g\left(e_{2}^{q}\right)=e_{2}^{0} \overline{e_{2}^{0}} e_{2}^{q} \overline{e_{1}^{0}} e_{1}^{1}, \\
& g\left(e_{1}^{i}\right)=e_{1}^{i+1}(i=0, \ldots, p-1), \\
& g\left(e_{2}^{i}\right)=e_{2}^{i+1}(i=0, \ldots, q-1) .
\end{aligned}
$$

(See Figure 10.)

\section{Subtemplates in branched covers}

The various cases of the Main Theorem all possess the same strategy of proof. Given the braid $b=b_{\left(n_{1}, \ldots, n_{k}\right)}$, we suspend the train track $G=G_{\left(n_{1}, \ldots, n_{k}\right)}$ 

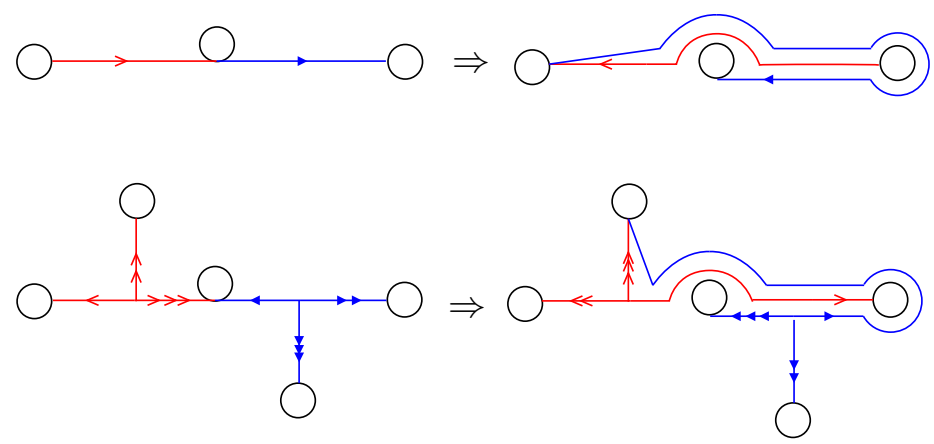

Figure 10. Top: $g_{(1,1)}$. Bottom: $g_{(2,2)}$.

by the train track map $g=g_{\left(n_{1}, \ldots, n_{k}\right)}$ to obtain a template $\mathcal{T}_{\left(n_{1}, \ldots, n_{k}\right)}$. We then use the branched covering method to obtain a template $\mathcal{U}_{\left(n_{1}, \ldots, n_{k}\right)}^{m}$ that captures the monodromy of the closure of the braid $b^{m}$. Since, in general, $\mathcal{U}_{\left(n_{1}, \ldots, n_{k}\right)}^{m}$ is much too complex to visualize, we focus on a particular subtemplate that we can show to be universal. In this section, we prove the technical results for restricting attention to appropriately simple subtemplates.

4.1. A normal form. We now derive the branched surface $\mathcal{T}_{(p, q)}$ obtained from $G_{(p, q)}$ by suspending $g_{(p, q)}$ from the previous section. We assume $p, q>$ 1. (Other cases are much simpler and are left to the reader.) Let $q_{\ell}, q_{r}$ be vertices of $S_{1}$ and $S_{2}$ of valence $p+1$ and $q+1$ respectively. They suspend to give the knots $k_{\ell}$ and $k_{r}$. Notice that the set of loop-edges in $S_{1} \cup S_{2}$ is a periodic orbit, say $A_{(p, q)}$, corresponding to the closure of $b_{(p, q)}$.

To visualize $\mathcal{T}_{(p, q)}$ as a template, we need to cut open $q_{\ell}, q_{r}$ and $A_{(p, q)}$, and flatten out the strips in some canonical way. See Figure 11.

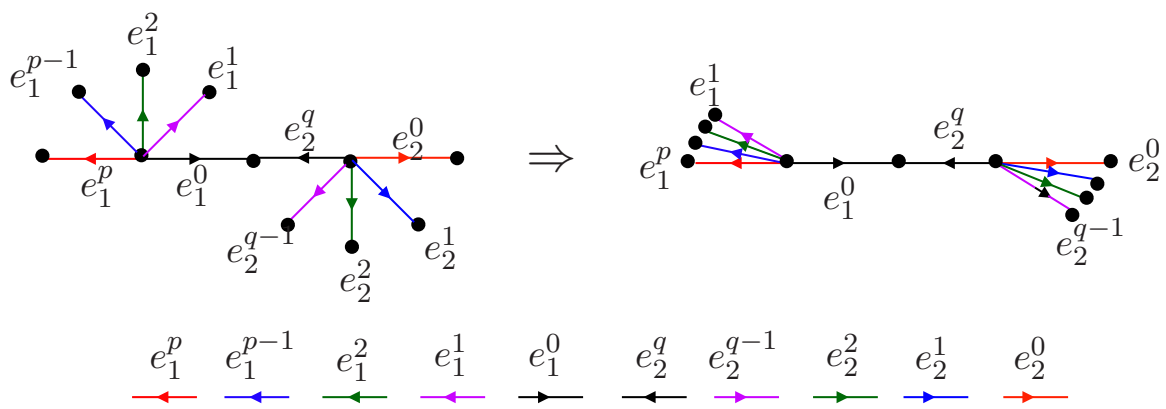

Figure 11. Flattening a pair of stars into strips. 
Recall that $g\left(e_{1}^{p}\right)=e_{1}^{0} \overline{e_{2}^{q}} e_{2}^{0}$. We split the edge $e_{1}^{p}$ into $E$ and $E^{\prime}$ so that $g(E)=e_{1}^{0} \overline{e_{2}^{q}}$ and $g\left(E^{\prime}\right)=e_{2}^{0}$. Split the edge $e_{2}^{q}$ into $F, F^{\prime}$ and $F^{\prime \prime}$ so that $g(F)=e_{2}^{0}, g\left(F^{\prime}\right)=\overline{e_{2}^{0}}$ and $g\left(F^{\prime \prime}\right)=e_{2}^{q} \bar{e}_{1}^{0} e_{1}^{1}$. For the template $\mathcal{T}_{(p, q)}$, we can use the symbols $x_{i=0 . p+1}$, and $y, z_{j=0 . . q+1}$ for the first and second stars respectively. The edge $e_{1}^{0}$ goes to $\mathrm{x}_{0}$, and the edge $e_{2}^{0}$ goes to $\mathrm{z}_{0}$, etc., and $e_{1}^{p}$ is split into $\mathrm{x}_{\mathrm{p}}$ and $\mathrm{x}_{\mathrm{p}+1}$ corresponding to $E$ and $E^{\prime}$, and $e_{2}^{q}$ split into $\mathrm{z}_{\mathrm{q}}, \mathrm{z}_{\mathrm{q}+1}, \mathrm{y}$ corresponding to $F, F^{\prime}$ and $F^{\prime \prime}$. We denote the left end point and right end point of the branch segment of the strip $\mathrm{x}$ by $p_{\ell}(\mathrm{x})$ and $p_{r}(\mathrm{x})$ respectively. Since the initial vertex of $e_{1}^{0}, e_{1}^{1}, \ldots, e_{1}^{p}$ is the same vertex $q_{\ell}$, we must abstractly identify (1) $p_{r}\left(\mathrm{x}_{1}\right), p_{r}\left(\mathrm{x}_{2}\right), \ldots, p_{r}\left(\mathrm{x}_{\mathrm{p}}\right)$ and $p_{\ell}\left(\mathrm{x}_{0}\right)$. In the same manner, we need to identify $(2) p_{r}\left(\mathrm{x}_{0}\right)$ and $p_{\ell}(\mathrm{y}),(3) p_{r}(\mathrm{y})$ and $p_{\ell}\left(\mathbf{z}_{\mathbf{q}+1}\right),(4) p_{r}\left(\mathbf{z}_{\mathbf{q}+1}\right)$ and $p_{\ell}\left(\mathbf{z}_{\mathbf{q}}\right),(5) p_{\ell}\left(\mathbf{z}_{0}\right), p_{\ell}\left(\mathbf{z}_{1}\right), \ldots, p_{\ell}\left(\mathbf{z}_{\mathbf{q}-1}\right)$ and $p_{r}\left(\mathbf{z}_{\mathbf{q}}\right)$, and $(6) p_{\ell}\left(\mathrm{x}_{\mathrm{p}}\right)$ and $p_{r}\left(\mathrm{x}_{\mathrm{p}+1}\right)$.

Upon suspension of the train track maps, the symbols $x_{i}, y$, and $z_{i}$ will correspond to portions of the branch lines, or, equivalently, to the exiting strips.

Henceforth we conveniently leave these boundary curves as distinct. When constructing subtemplates of this branched surface, we are careful never to use these edges within the subtemplates. In this way, we do not need to worry about the changes to the periodic orbits set we have made.

The normal form of $\mathcal{T}_{(p, q)}$ appears in cartoon form as in Figure 12.

To construct $\mathcal{U}_{(p, q)}^{m}$, the template for the $m$-fold branched cover of $\mathcal{T}_{(p, q)}$, we must peel off a parallel copy of $\beta$ and cut $\mathcal{T}_{(p, q)}$ by a spanning disc for this unknot: compare the figure-eight knot case in Figure 4 . Thus, the fundamental domain of $\mathcal{U}_{(p, q)}^{m}$ depends on the linking properties of $\beta$ with $\mathcal{T}_{(p, q)}$.

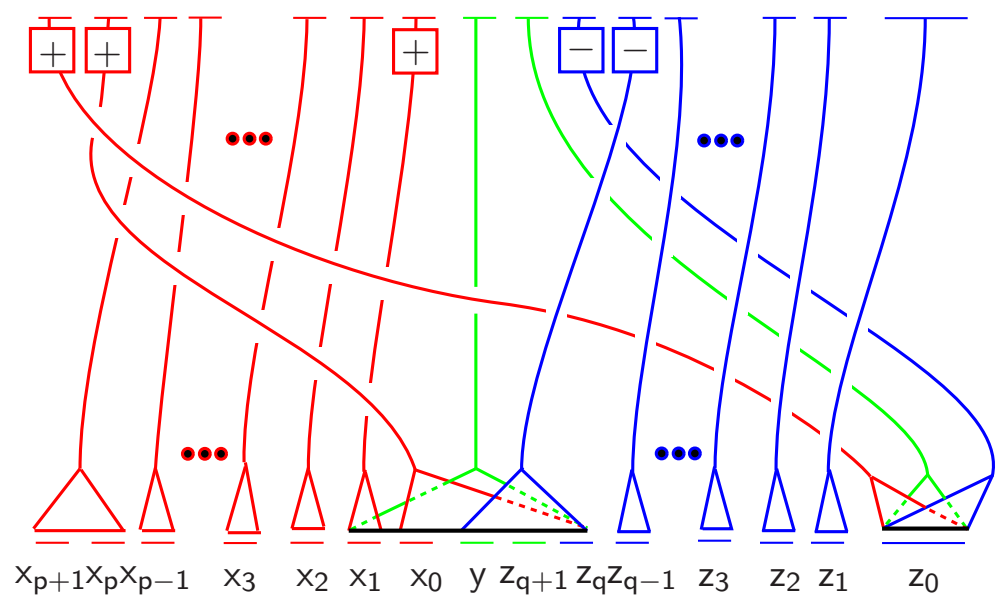

Figure 12. The normal form for the template $\mathcal{T}_{(p, q)}$. 


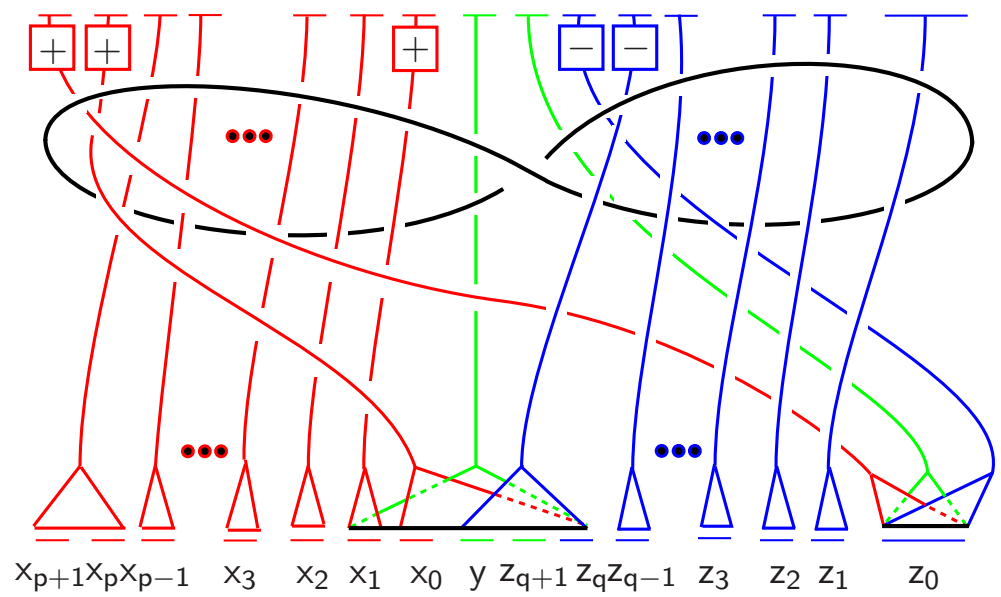

Figure 13. A parallel copy of the closed braid $\beta$ links certain strips of $\mathcal{T}_{(p, q)}$.

Lemma 4.1. Let $\beta$ be the closure of $b_{(p, q)}$ and $\beta^{\prime}$ a parallel copy. Then $\beta^{\prime}$ links $\mathcal{T}_{(p, q)}$ as in Figure 13.

Proof. Note that $g_{\left(n_{1}, n_{2}\right)}$ has three fixed points $q_{\ell}, q_{c}, q_{r}$, where $q_{\ell}$ and $q_{r}$ are as above and $q_{c} \in e_{2}^{q}$ (see Lemma 3.6). These suspend to give the three unknots $k_{\ell}, k_{c}$, and $k_{r}$. It is clear from the image of the train track (see Figures 10 and 14) that the fixed point $q_{c}$ is "in back" of the image of all the other edges, and that consequently the suspension of this fixed point,
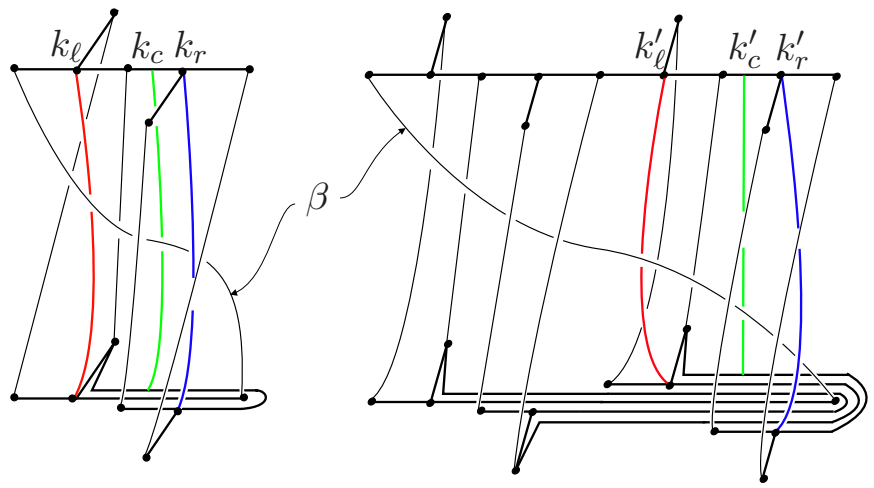

Figure 14. Left: $p=q=2$. Right: $k=4$ and $n_{1}=n_{2}=$ $n_{3}=n_{4}=2$. 
$k_{c}$, is an unknot that has twist zero and is separable from all other orbits on the template. Hence, $\ell k\left(k_{c}, \beta\right)=0$. Since the train track map rotates each star about its central vertex with opposite orientations, it is easy to see that $\ell k\left(k_{\ell}, \beta\right)=+1$ and $\ell k\left(k_{r}, \beta\right)=-1$ (see Figure 14, left).

Since the strip y contains $k_{c}$ and $\ell k\left(k_{c}, \beta\right)=0$, y and $\beta$ are linked as in Figure 14. Because of the way we unfolded the star in Figure 11, the way that $\mathrm{z}_{\mathbf{q}+1}$ and $\beta$ are linked is the same as for $\mathrm{y}$ and $\beta$.

The edges $e_{1}^{0}, \ldots, e_{1}^{p}$ were all split off $q_{\ell}$, and $\ell k\left(k_{\ell}, \beta\right)=+1$. Hence $\mathrm{x}_{0}, \ldots, \mathrm{x}_{\mathrm{p}}, \mathrm{x}_{\mathrm{p}+1}$ and $\beta$ are linked as in Figure 14. Since the edges $e_{2}^{0}, \ldots, e_{2}^{q-1}$, $F\left(\subset e_{2}^{q}\right)$ were also split off $q_{r}$ and $\ell k\left(k_{r}, \beta\right)=-1$, the strips $\mathrm{z}_{0}, \ldots, \mathrm{z}_{\mathbf{q}-1}, \mathrm{z}_{\mathbf{q}}$ and $\beta$ are linked as in Figure 14. This completes the proof.

By Lemma 4.1, the fundamental domain of $\mathcal{U}_{(p, q)}^{m}$ is as in Figure 15, where we have cut along a spanning disc of $\beta$. To form $\mathcal{U}_{(p, q)}^{m}$, take $m$ copies of this fundamental domain joined cyclically end-to-end: see, e.g., Figure 17.

4.2. Subtemplates. The (very complicated) normal form $\mathcal{U}_{\left(n_{i}, n_{i+1}\right)}^{m}$ is tame compared to the full template $\mathcal{U}_{\left(n_{1}, \ldots, n_{k}\right)}^{m}$. Fortunately, we do not need to consider the most general case.

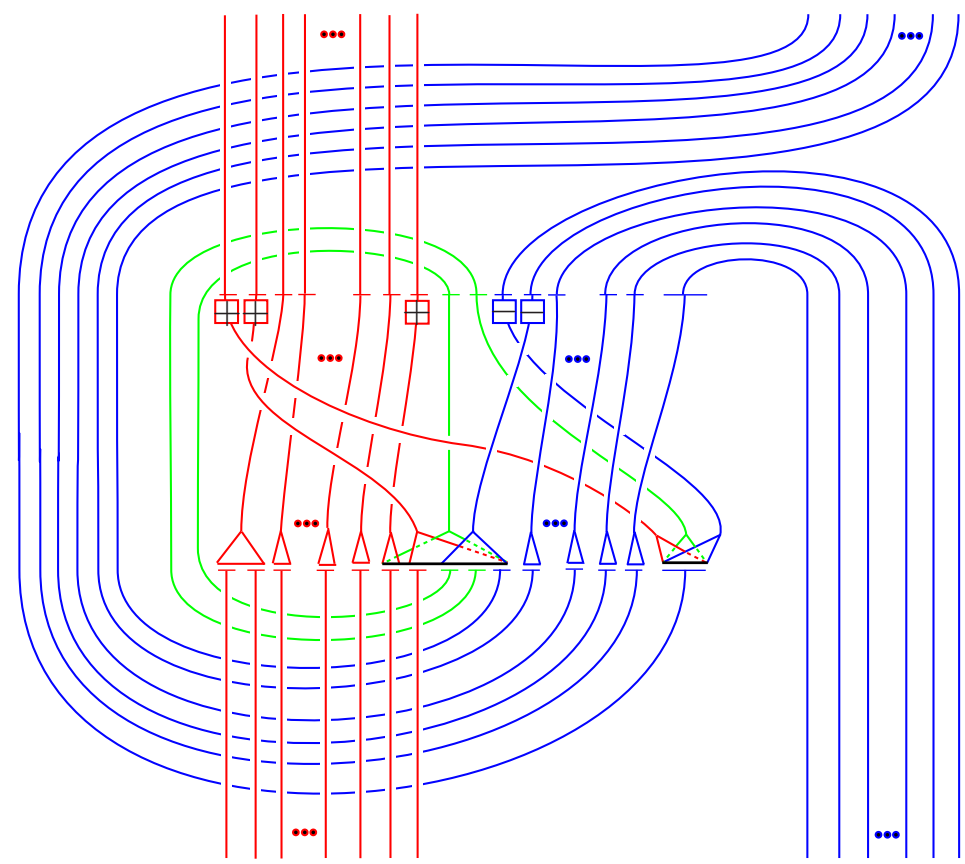

Figure 15. The fundamental domain of $\mathcal{U}_{(p, q)}^{m}$ (see Figure 4). 
Lemma 4.2. $\mathcal{T}_{\left(n_{i}, n_{i+1}\right)}($ if $i$ is odd) or its mirror image (if $i$ is even) is a subtemplate of $\mathcal{T}_{\left(n_{1}, \ldots, n_{k}\right)}$.

Proof. We prove this result on the level of the train track (by subdividing and removing extraneous portions), and then suspend this to obtain the result for subtemplates. We only prove the case $n_{i}, n_{i+1}>1$, for other cases are similar. Let $G=G_{\left(n_{1}, \ldots, n_{k}\right)}$ and $g=g_{\left(n_{1}, \ldots, n_{k}\right)}$. For the proof of the lemma, it is enough to show that there exist subdivisions $I_{2} I_{1}=e_{i}^{n_{i}}$, $J_{3} J_{2} J_{1}=e_{i+1}^{n_{i+1}}$ of $G$ that satisfy the following:

Remove $I_{2}$ and $J_{2}$ from $S_{i}$ and $S_{i+1}$ respectively. Denote by $\left(S_{i} \cup S_{i+1}\right)^{\prime}$ the graph obtained from $\left(S_{i} \cup S_{i+1}\right)$ by identifying the right end point of $J_{3}$ and the left end point of $J_{1}$. Then $g\left(\left(S_{i} \cup S_{i+1}\right)^{\prime}\right)=g_{i+1}\left(g_{i}\left(S_{i} \cup S_{i+1}\right)\right)$. (See Figure 16.)
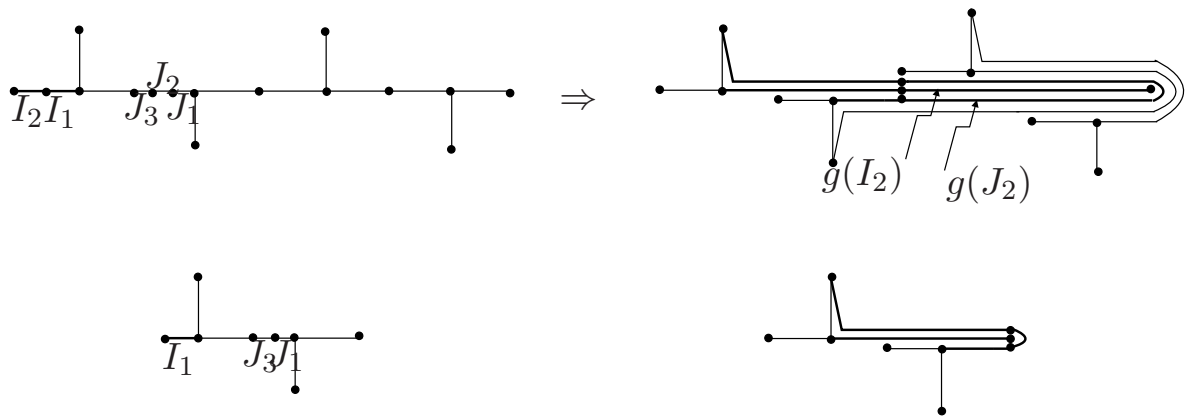

Figure 16. Top: $g=g_{(2,2,1)}$. Lower left: $\left(S_{1} \cup S_{2}\right)^{\prime}$. Lower right: $g\left(\left(S_{1} \cup S_{2}\right)^{\prime}\right)$, which equals $g_{2}\left(g_{1}\left(S_{1} \cup S_{2}\right)\right)$.

We demonstrate the case $i=1$, the proof for other cases being similar. Denote by $c_{i}$ the following edges of the train track: $c_{i}=e_{i}^{0}$ if $n_{i}=1$ and $c_{i}=\overline{e_{i}^{n_{i}}} e_{i}^{0}$ if $n_{i}>1$. By induction on $k$, one demonstrates that

$$
g\left(e_{1}^{n_{1}}\right)=e_{1}^{0} c_{2} \cdots c_{k}, \quad g\left(e_{2}^{n_{2}}\right)=e_{2}^{0} c_{3} \cdots c_{k-1} c_{k} \overline{c_{k}} \overline{c_{k-1}} \cdots \overline{c_{2}} \overline{e_{1}^{0}} e_{1}^{1},
$$

and $g(e)=g_{2}\left(g_{1}(e)\right)$ for $e \in S_{1} \cup S_{2} \backslash\left\{e_{1}^{n_{1}}, e_{2}^{n_{2}}\right\}$. Take subdivisions $I_{2} I_{1}=e_{1}^{n_{1}}$ and $J_{3} J_{2} J_{1}=e_{2}^{n_{2}}$ such that $g\left(I_{1}\right)=e_{1}^{0} c_{2}, g\left(J_{3}\right)=e_{2}^{0}$, and $g\left(J_{1}\right)=\overline{c_{2}} \overline{e_{1}^{0}} e_{1}^{1}$. Since $g\left(I_{1}\right)=g_{2}\left(g_{1}\left(e_{1}^{n_{1}}\right)\right), g\left(J_{3}\right) g\left(J_{1}\right)=g_{2}\left(g_{1}\left(e_{2}^{n_{2}}\right)\right)$ and $g(e)=g_{2}\left(g_{1}(e)\right)$ for $e \in S_{1} \cup S_{2} \backslash\left\{e_{1}^{n_{1}}, e_{2}^{n_{2}}\right\}$, we have $g\left(\left(S_{1} \cup S_{2}\right)^{\prime}\right)=g_{2}\left(g_{1}\left(S_{1} \cup S_{2}\right)\right)$.

Lemma 4.3. $\mathcal{U}_{\left(n_{i}, n_{i+1}\right)}^{m}$ (if $i$ is odd) or its mirror image (if $i$ is even) is a subtemplate of $\mathcal{U}_{\left(n_{1}, \ldots, n_{k}\right)}^{m}$. 
Proof. We consider the case of $i$ odd and $n_{i}, n_{i+1}>1$; the other cases share identical proofs. The key step in this lemma is to determine the linking properties of $\beta$ with the subtemplate obtained from Lemma 4.2.

Note that $g_{\left(n_{1}, \ldots, n_{k}\right)}$ has three corresponding fixed points $q_{\ell}^{\prime}, q_{c}^{\prime}, q_{r}^{\prime}$, where $q_{\ell}^{\prime}$ and $q_{r}^{\prime}$ are $\left(n_{i}+1\right)$ vertex and $\left(n_{i+1}+1\right)$ vertex of $S_{i}$ and $S_{i+1}$ respectively, and $q_{c}^{\prime} \in e_{i+1}^{n_{i+1}}$. Denote by $k_{\ell}^{\prime}, k_{c}^{\prime}, k_{r}^{\prime}$ the three unknots given by suspending these points. Let $\beta$ and $\beta^{\prime}$ be the closures of $b_{\left(n_{1}, n_{2}\right)}$ and $b_{\left(n_{1}, \ldots, n_{k}\right)}$ respectively. As in the proof of Lemma 4.1, one shows that $\ell k\left(k_{\ell}^{\prime}, \beta^{\prime}\right)=\ell k\left(k_{\ell}, \beta\right)=+1$, $\ell k\left(k_{c}^{\prime}, \beta^{\prime}\right)=\ell k\left(k_{c}, \beta\right)=0$, and $\ell k\left(k_{r}^{\prime}, \beta^{\prime}\right)=\ell k\left(k_{r}, \beta\right)=-1$, see Figure 14 , rigth. This, together with Lemma 4.2, implies Lemma 4.3.

\section{The main theorems}

We now have assembled all the ingredients to prove the main theorems.

5.1. Main Theorem (c). We begin with the easiest and least general of the three Main Theorems. A proof can be obtained implicitly by combining Lemma 4.3 with a geometric argument from [14, pp. 444-5]. However, to set a pattern for the proofs of Main Theorems (a) and (b), we give a different proof of (c).

Theorem 5.1. Let $b=b_{\left(n_{1}, \ldots, n_{k}\right)}$ where $n_{j}=n_{j+1}=1$ for some $j$. Then the closure of $b^{m}$ is a knot/link in $S^{3}$ with the universal fibration property for every $m \geq 2$.

Proof. From Lemma 4.3, we may restrict attention to the subtemplate $\mathcal{U}_{(1,1)}^{m}$ within $\mathcal{U}_{\left(n_{1}, \ldots, n_{k}\right)}^{m}$. The template $\mathcal{T}_{(1,1)}$ is given in cartoon form in Figure 4, along with a representation of the fundamental domain of $\mathcal{U}_{(1,1)}^{m}$. Our strategy is to identify three unknots on $\mathcal{U}_{(1,1)}^{m}-\kappa, \kappa^{\prime}$, and $\kappa^{\prime \prime}$ - to which we can apply Theorem 2.4 .

Specifically, using the symbolic representation of the orbits as periodic words, let $\kappa=(\mathrm{y}), \kappa^{\prime}=\left(\mathrm{x}^{\mathrm{m}}\right)$, and $\kappa^{\prime \prime}=\left(\mathrm{z}^{\mathrm{m}}\right)$. Recall that $\left(\mathrm{x}^{\mathrm{m}}\right)$ means to follow the periodic orbit that traverses the $\mathrm{x}$-strip $m$ times. While there is an ambiguity in the symbolic representation (in terms of which fundamental domain the symbol is on), the only choice is for (y), and by cyclic symmetry, the result is independent of the choice.

It is trivial to see from Figure 17, left, that these three curves are separable unknots, and that $\kappa$ has zero twist, $\kappa^{\prime}$ has positive twist, and $\kappa^{\prime \prime}$ has negative twist. The only other ingredient needed to invoke Theorem 2.4 is that the three orbits meet at a branchline as in Figure 3. This is not the case, since the strip $z$ does not fully cover the branchline. To remedy this, we replace $\left(x^{m}\right)$ with $\left(y x^{m}\right)$. This orbit is still a separable unknot (using [13, Proposition 3.1.19]) whose (already positive) twist has increased by two and now passes through the triple branchline in precisely the manner of Figure 3. Hence, by Theorem $2.4, \mathcal{U}_{(1,1)}^{m}$ is universal. 

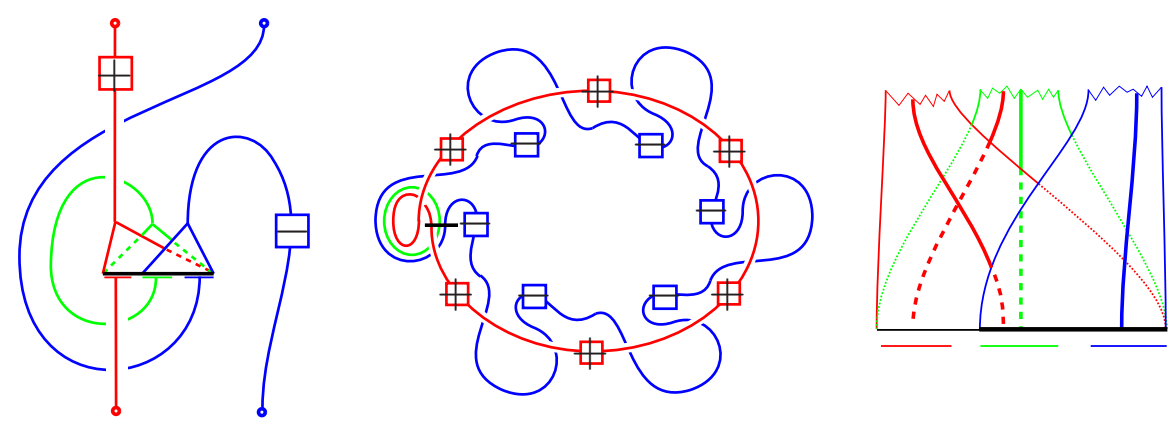

Figure 17. Left: a cartoon representation of the fundamental domain of $\mathcal{U}_{(1,1)}^{m}$. Center: the unknots $\kappa, \kappa^{\prime}$, and $\kappa^{\prime \prime}$ in the case $m=6$. Right: all three orbits pass through the same branch line.

5.2. Main Theorem (b). The following theorem allows us to completely classify the fibrations of 2-bridge knot complements in the next section.

Theorem 5.2. The closure of $\left(b_{\left(n_{1}, \ldots, n_{k}\right)}\right)^{2}$ has the universal fibration property.

Proof. To prove Theorem 5.2, it suffices to show that $\mathcal{U}_{(p, q)}^{2}$ is universal for all $p$ and $q$. We assume $p, q>1$, the other cases being both simpler and similar to Main Theorem (c). The strategy, as in the previous theorem, is to find three well-chosen unknots $\kappa, \kappa^{\prime}, \kappa^{\prime \prime}$ on $\mathcal{U}_{(p, q)}^{2}$.

As before, choose $\kappa:=(\mathrm{y})^{\infty}$ the untwisted unknot separable from all other orbits on the template. For $p$ odd (resp. even), define

$$
\kappa^{\prime}:=\left(\mathrm{yx}_{0} \mathrm{x}_{1} \mathrm{x}_{2} \cdots \mathrm{x}_{\mathrm{p}-1} \mathrm{x}_{\mathrm{p}}\right) \quad\left(\text { resp. } \kappa^{\prime}:=\left(\mathrm{yx}_{1} \mathrm{x}_{2} \cdots \mathrm{x}_{\mathrm{p}-1} \mathrm{x}_{\mathrm{p}}\right)\right)
$$

(see Figure 18). For $q$ odd (resp. even), define

$$
\kappa^{\prime \prime}:=\left(\mathrm{yz}_{\mathrm{q}} \mathrm{z}_{0} \mathrm{z}_{1} \cdots \mathrm{z}_{\mathrm{q}-1}\right) \quad\left(\text { resp. } \kappa^{\prime \prime}:=\left(\mathrm{yz}_{\mathrm{q}+1} \mathrm{z}_{0} \mathrm{z}_{1} \cdots \mathrm{z}_{\mathrm{q}-1}\right)\right)
$$

(see Figure 19). Note that there is an ambiguity as to which fundamental domain the orbit segment $y$ of $\kappa^{\prime}$ and $\kappa^{\prime \prime}$ passes through. We specify as follows: take $\kappa^{\prime}$ and $\kappa^{\prime \prime}$ such that the orbit segment $y$ passes through the fundamental domain where $\kappa$ lies. The figures imply that these are separable unlinked unknots with $\tau(\kappa)=0, \tau\left(\kappa^{\prime}\right)>0$, and $\tau\left(\kappa^{\prime \prime}\right)<0$. These unknots $\kappa, \kappa^{\prime}$ and $\kappa^{\prime \prime}$ pass through the triple branchline as in Figure 20. Hence, by Theorem 2.4, $\mathcal{U}_{(p, q)}^{2}$ is universal.

5.3. Main Theorem (a). For part (a) of the Main Theorem - that the universal fibration property holds for $m$ sufficiently large - we give a more precise formulation. 

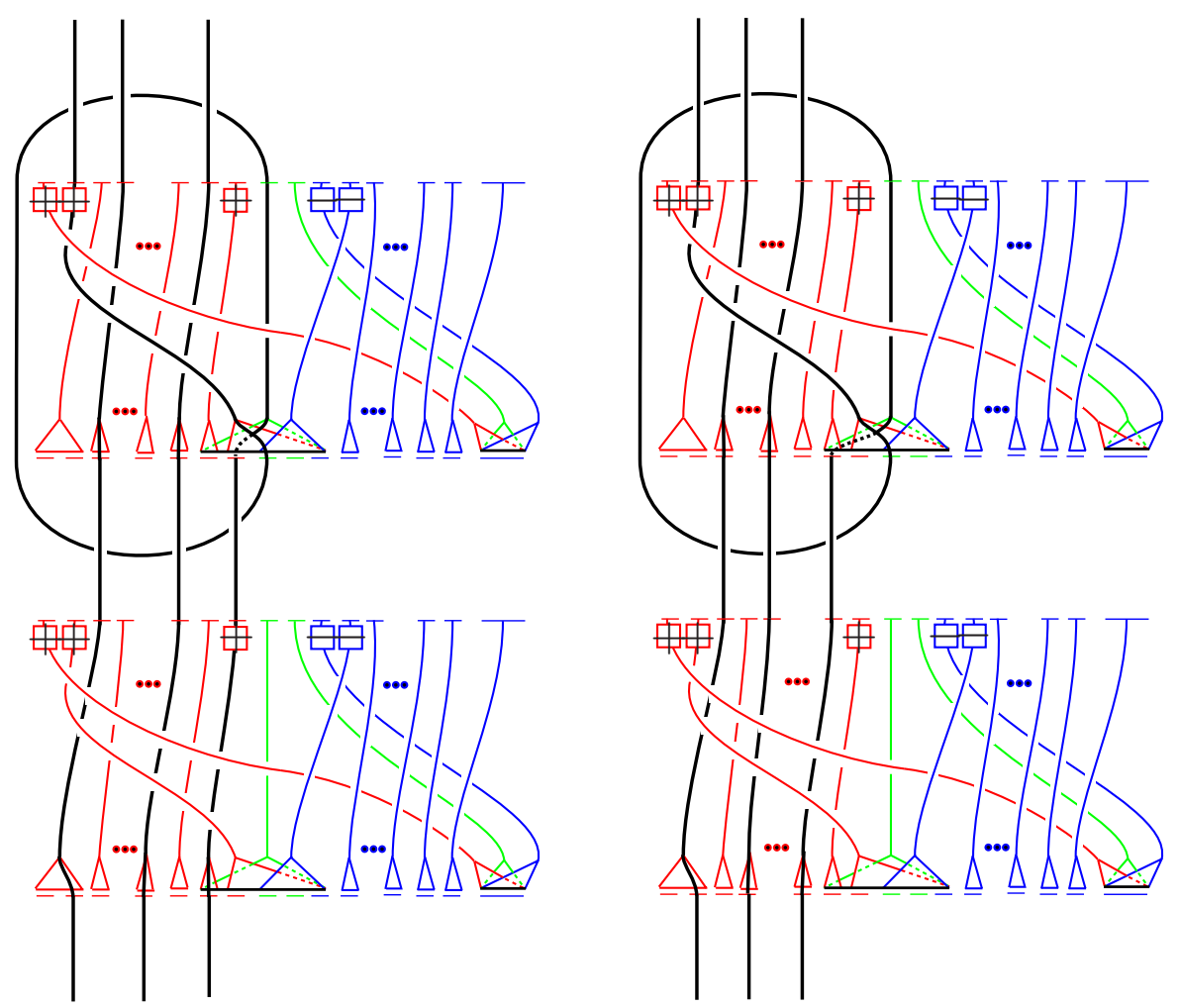

Figure 18. The orbit $\kappa^{\prime}$ in Main Theorem (b), when $p$ is odd (left) or even (right).

Theorem 5.3. Let $N:=\min _{i}\left(\max \left\{n_{i}, n_{i+1}\right\}\right)$. The closure of $\left(b_{\left(n_{1}, \ldots, n_{k}\right)}\right)^{m}$ has the universal fibration property for all $m \geq N(N-1)$.

Proof. By Lemma 4.3, it is enough to show that $\mathcal{U}_{(p, q)}^{m}$ is universal for each $m \geq N(N-1)$, where $N=\max \{p, q\}$. As before, we assume $p$ and $q$ greater than 1 .

Let $m=p t^{\prime}+s^{\prime}=q t^{\prime \prime}+s^{\prime \prime}$ be Euclidean integer factorizations of $m$; hence $0 \leq s^{\prime}<p$ and $0 \leq s^{\prime \prime}<q$. As $m \geq N(N-1)$, it follows that $t^{\prime} \geq s^{\prime}$ and $t^{\prime \prime} \geq s^{\prime \prime}$. Consider the following three periodic orbits on $\mathcal{U}_{(p, q)}^{m}$ :

$$
\begin{aligned}
\kappa & :=(\mathrm{y}) \quad \kappa^{\prime}:=\left(\left(\mathrm{yx}_{1} \cdots \mathrm{x}_{\mathrm{p}}\right)^{\mathrm{t}^{\prime}-\mathrm{s}^{\prime}}\left(\mathrm{x}_{0} \mathrm{x}_{1} \cdots \mathrm{x}_{\mathrm{p}}\right)^{\mathrm{s}^{\prime}}\right) \\
\kappa^{\prime \prime} & :=\left(\left(\mathrm{z}_{\mathrm{q}+1} \mathrm{z}_{0} \mathrm{z}_{1} \cdots \mathrm{z}_{\mathrm{q}-1}\right)^{\mathrm{t}^{\prime \prime}-\mathrm{s}^{\prime \prime}}\left(\mathrm{z}_{\mathrm{q}} \mathrm{z}_{0} \mathrm{z}_{1} \cdots \mathrm{z}_{\mathrm{q}-1}\right)^{\mathrm{s}^{\prime \prime}}\right) .
\end{aligned}
$$

The orbit $\kappa$ is, as before, an untwisted unknot lying within one fundamental domain of $\mathcal{U}_{(p, q)}^{m}$. The orbit $\kappa^{\prime}$ passes through $p\left(t^{\prime}-s^{\prime}\right)+(p+1) s^{\prime}=p t^{\prime}+s^{\prime}=$ $m$ fundamental domains of $\mathcal{U}_{(p, q)}^{m}$. Except for the occasional excursions about 

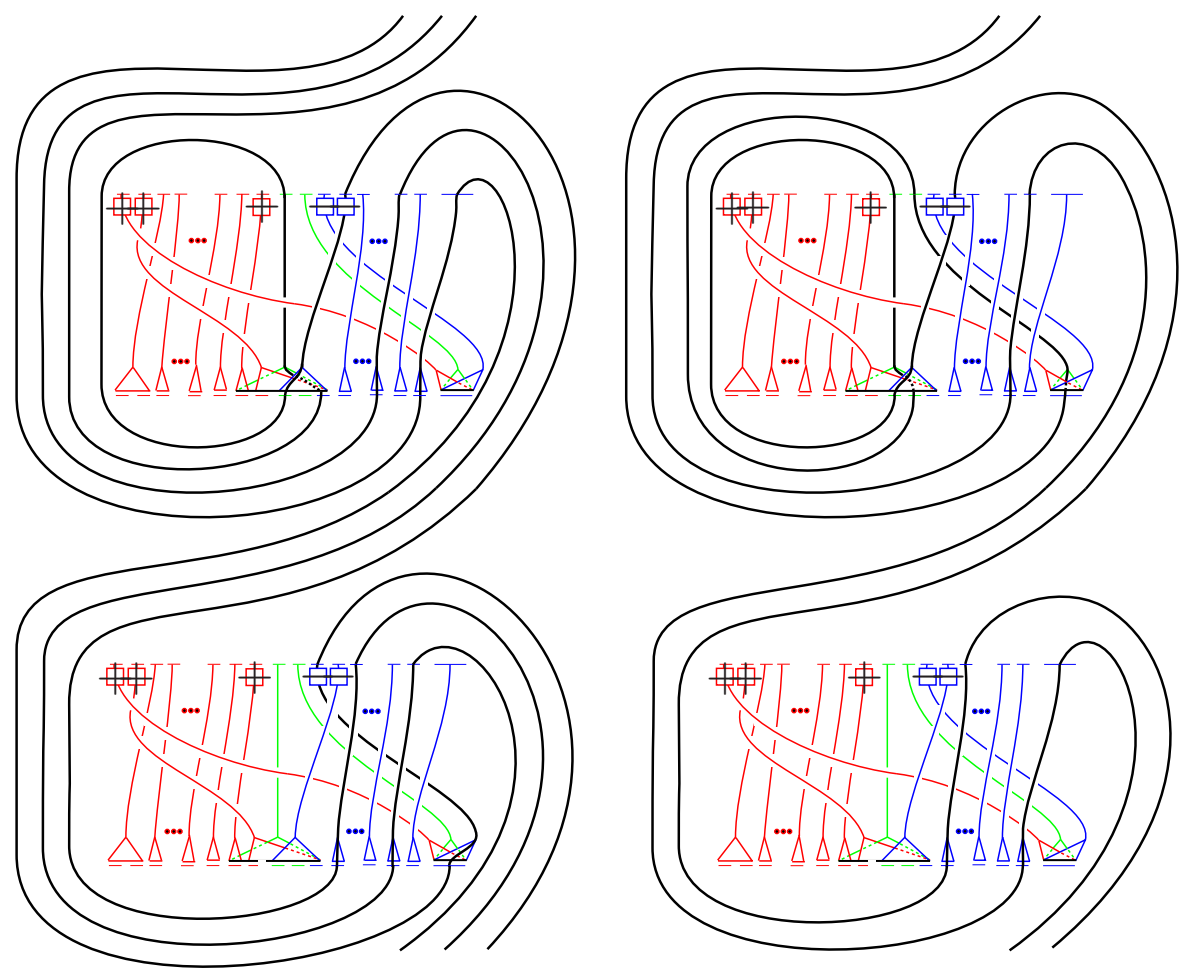

Figure 19. The orbit $\kappa^{\prime \prime}$ in Main Theorem (b), when $q$ is odd (left) or even (right).
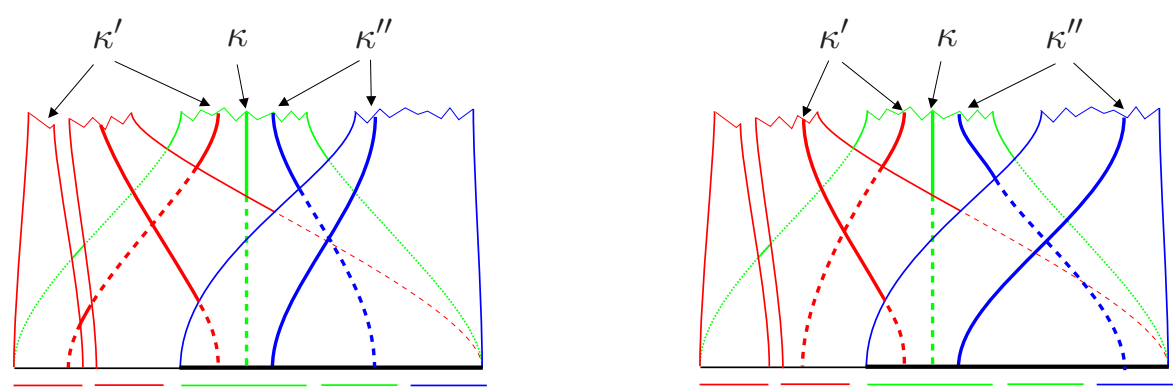

Figure 20. A closeup of the branch line in the proof of Main Theorem (b), when $p$ and $q$ are even (left) or odd (right).

the y orbit (which do not change the knot type), the orbit $\kappa^{\prime}$ can be thought of as a 1-strand braid about the braid axis of $\mathcal{U}_{(p, q)}^{m}$ : it is therefore an unknot. All twisting in the $\mathrm{x}$-strips is of a positive nature, so that $\kappa^{\prime}$ is 
an unknot with positive twist. A similar argument shows that $\kappa^{\prime \prime}$ passes through $q\left(t^{\prime \prime}-s^{\prime \prime}\right)+(q+1) s^{\prime \prime}=m$ fundamental domains and is an unknot with negative twist separable from $\kappa^{\prime}$.

These orbits are a triple of separable unknots satisfying the conditions of Theorem 2.4, as illustrated in Figure 21. Hence $\mathcal{U}_{(p, q)}^{m}$ is universal.

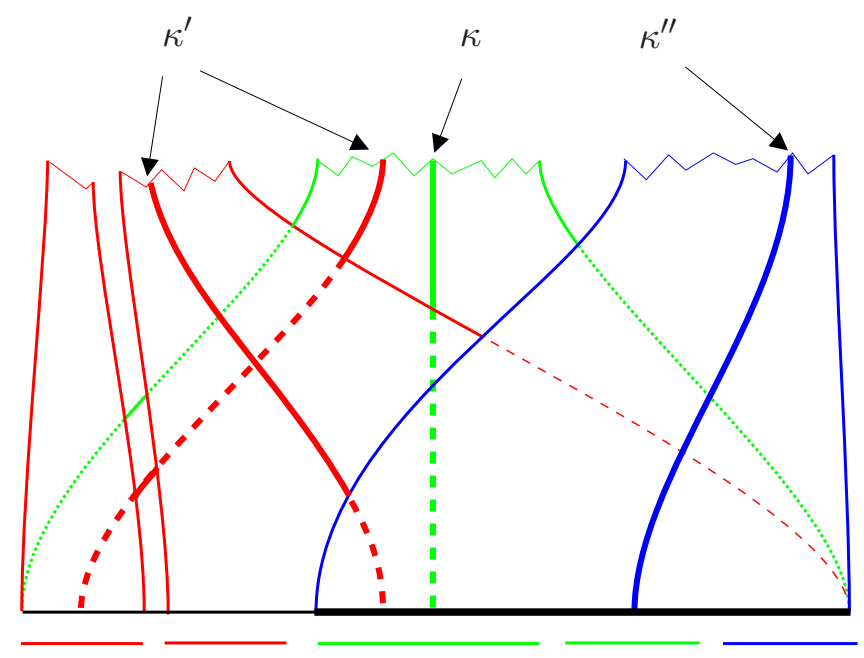

Figure 21. A closeup of the branch line in the proof of Main Theorem (a).

\section{Concluding remarks}

We have displayed a large family of fibred knots/links whose fibrations force all knot and link types in the complement. It remains an open question how large this family can be:

Question 6.1. Is every fibred knot/link of pseudo-Anosov type universally fibred?

We conclude this paper with a few remarks on this question.

6.1. 2-bridge knots. Recall that a two-bridge knot is one that can be isotoped so as to have precisely four critical points (two minima and two maxima) with respect to the standard height function in $\mathbb{R}^{3}$. We can answer Question 6.1 affirmatively in this category.

Corollary 6.2. Every fibred nontorus two-bridge knot has the universal fibration property. 
Proof. The work of Gabai and Kazez [12] explicitly shows that the class of fibred 2-bridge knots is precisely that class of knots whose fibres are a chain of plumbed Hopf bands, and that the monodromy is of pseudo-Anosov type if and only if the Hopf bands are not all of the same sign, which is to say, if and only if it is not a torus knot. We claim that such a chain of plumbed Hopf bands has a closed braid form of $\left(b_{\left(n_{1}, \ldots, n_{k}\right)}\right)^{2}$. The proof is inductive on the number of Hopf bands and illustrated in Figure 22. The key step is noting that the fibre for the closure of $\left(b_{\left(n_{1}, \ldots, n_{k}\right)}\right)^{2}$ is a stack of discs attached by pairs of half-twisted bands: this is precisely a chain of plumbed Hopf bands. From Theorem 5.2, we have that this knot must be universally fibred.
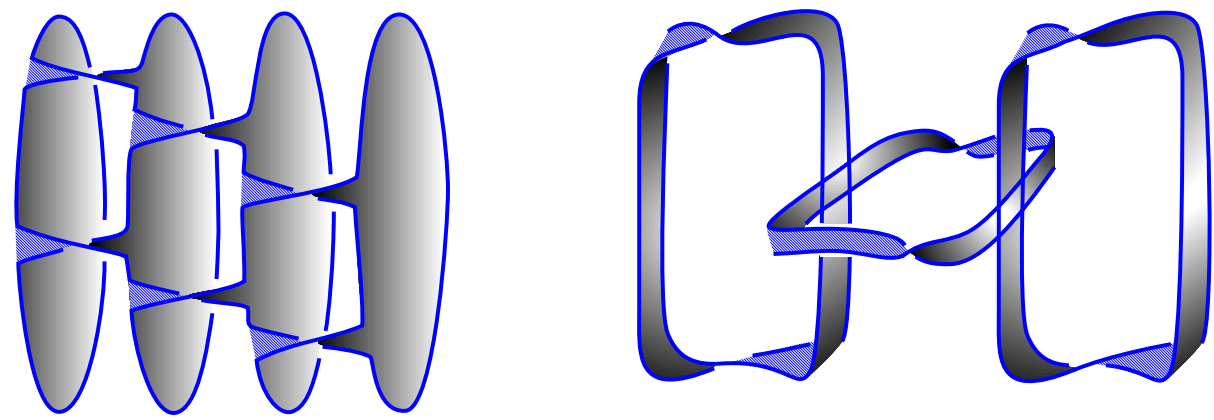

Figure 22. Plumbings of Hopf bands (right) can be arranged as a closed braid of the form $\left(b_{n_{1}, \ldots, n_{k}}\right)^{2}$ (left).

6.2. Connected sums. We can enlarge the class of universally fibred knots considerably by taking connected sums.

Proposition 6.3. The connected sums of any fibred knot/link with any universally fibred knot/link is universally fibred.

Proof. In [11, Corollary 1.4.], it is shown that the Murasugi sum of fibre surfaces is "natural". Specifically, if $K_{i}$ is a fibred knot/link $(i=1,2)$ with fibre surface $\Sigma_{i}$ and monodromy $\Phi_{i}$ satisfying $\left.\Phi_{i}\right|_{\partial \Sigma_{i}}=i d$, then the Murasugi sum of $\Sigma_{1}$ and $\Sigma_{2}$ along a $2 k$-gon $D$ common to $\Sigma_{1}$ and $\Sigma_{2}$ is fibre surface and has monodromy $\widetilde{\Phi}_{1} \circ \widetilde{\Phi}_{2}$, where $\widetilde{\Phi}_{i}$ is equal to $\Phi_{i}$ on $\Sigma_{i}$ and the identity map elsewhere.

Even more importantly, the proof of this result uses vector fields transverse to the fibration to achieve the result. Thus, we actually have that a transverse vector field of the Murasugi sum decomposes naturally.

The simplest kind of Murasugi sum (where the attachment is along a 2-gon in the spanning surfaces) is precisely the connected sum. Choose 
the monodromy $\Phi_{1}$ of the universally fibred knot to be the pseudo-Anosov representative, isotoped so as to be the identity map on the the boundary. Likewise, choose the monodromy $\Phi_{2}$ to be the Thurston canonical form isotoped to be the identity on the boundary. The composition monodromy on the connected (in this case, Murasugi) sum is easily isotoped to have an invariant curve separating the universal pseudo-Anosov component from the Thurston form of $\Phi_{2}$. Thus, the theorem of Asimov and Franks implies that any transverse vector field must have at least this set of periodic orbits all knots and links.

As a corollary of the proof, we see that in certain instances, more general Murasugi sums (e.g., plumbing) can preserve the universal fibration property as well. One would need to control:

(1) the dynamics on the attaching region, to preserve the pseudo-Anosov property of the monodromy, and

(2) the location of the attaching region, so that it does not interfere with the universal subtemplate.

6.3. Too-twisted monodromies. We believe that there are pseudo-Anosov fibred knots or links whose monodromies are too "twisted" to admit a universal template in the suspension. Our proposed example stems from the fact that the proof of Main Theorem (c) requires a sufficiently high order branched cover.

Conjecture 6.4. For $p$ and $q$ sufficiently large, the closure of $\left(b_{(p, q)}\right)^{3}$ is not universally fibred.

Our reason for conjecturing the nonuniversality is the difficulty in finding enough unknotted orbits on which to build a universal subtemplate.

\section{References}

[1] D. Asimov and J. Franks, Unremovable closed orbits, in 'Geometric Dynamics' (ed. J. Palis), Lect. Notes in Math., 1007, Springer, Berlin, 1983, 22-29, MR 0730260 (86a:58083), Zbl 0521.58047.

[2] M. Bestvina and M. Handel, Train-tracks for surface homeomorphisms, Topology, 34(1) (1994), 109-140, MR 1308491 (96d:57014), Zbl 0837.57010.

[3] J. Birman, A representation theorem for fibered knots and their monodromy maps, in 'Topology of Low-Dimensional Manifolds' (Proc. Second Sussex Conf., Chelwood Gate, 1977), Lecture Notes in Math., 722, Springer, Berlin, 1979, 1-8, MR 0547448 (80k:57007), Zbl 0406.57004.

[4] J. Birman and R. Williams, Knotted periodic orbits in dynamical systems, I. Lorenz's equations, Topology, 22(1) (1983), 47-82, MR 0682059 (84k:58138), Zbl 0507.58038.

[5] J. Birman and R. Williams, Knotted periodic orbits in dynamical systems, II: Knot holders for fibered knots, Contemp. Math., 20 (1983), 1-60, MR 0718132 (86a:58084), Zbl 0526.58043. 
[6] A. de Carvalho and T. Hall, Braid forcing and star-shaped train tracks, Topology, 43(2) (2004), 247-287.

[7] J. Casasayas, J. Martinez Alfaro and A. Nunes, Knots and links in integrable Hamiltonian systems, J. Knot Theory Ramifications, 7(2) (1998), 123-153, MR 1618908 (99f:58111), Zbl 0899.57005.

[8] J. Etnyre and R. Ghrist, Gradient flows within plane fields, Comment. Math. Helv., 74 (1999), 507-529, MR 1730655 (2000k:37030), Zbl 0964.37023.

[9] A. Fomenko and T.Z. Nguyen, Topological classification of integrable nondegenerate Hamiltonians on isoenergy three-dimensional spheres, in 'Topological Classification of Integrable Systems' (A. Fomenko ed.), Adv. Soviet Mathematics, 6, Amer. Math. Soc., Providence, RI, 1991, 267-296, MR 1141227 (93a:58060), Zbl 0744.58033.

[10] J. Franks, Knots, links, and symbolic dynamics, Ann. of Math., 113 (1981), 529-552, MR 0621015 (83h:58074), Zbl 0469.58013.

[11] D. Gabai, The Murasugi sum is a natural geometric operation, II, Contemp. Math., 44 (1985), 93-100, MR 0813105 (87f:57003), Zbl 0524.57004.

[12] D. Gabai and W. Kazez, Pseudo-Anosov maps and surgery on fibred 2-bridge knots, Topology Appl., 37 (1990), 93-100, MR 1075377 (91j:57005), Zbl 0714.57004.

[13] R. Ghrist, P. Holmes and M. Sullivan, Knots and Links in Three-Dimensional Flows, Lect. Notes in Math., 1654, Springer, Berlin, 1997, MR 1480169 (98i:58199), Zbl 0869.58044.

[14] R. Ghrist, Branched two-manifolds supporting all links, Topology, 36(2) (1997), 423448, MR 1415597 (98b:57009), Zbl 0869.57007.

[15] D. Goldsmith, Symmetric fibered links, in 'Knots, Groups, and 3-Manifolds', Ann. of Math. Studies, 84, Princeton Univ. Press, Princeton, 1975, 3-23, MR 0380766 (52 \#1663), Zbl 0331.55001.

[16] P. Melvin and N. Tufillaro, Templates and framed braids, Phys. Rev. A, 44(6) (1991), 3419-3422, MR 1130087 (92m:57013).

[17] H. Morton and M. Rampichini, Mutual braiding and the band presentation of braid groups, in 'Knots in Hellas '98 (Delphi)', 335-346, Ser. Knots Everything, 24, World Sci. Publishing, River Edge, NJ, 2000, MR 1865715 (2002h:57013), Zbl 0998.57014.

[18] R. Penner and J. Harer, Combinatorics of Train Tracks, Ann. of Math. Studies, 125, Princeton Univ. Press, Princeton, 1992, MR 1144770 (94b:57018), Zbl 0765.57001.

[19] D. Rolfsen, Knots and Links, Publish or Perish Press, 1976, MR 0515288 (58 \#24236), Zbl 0339.55004.

[20] W. Thurston, On the geometry and dynamics of diffeomorphims of surfaces, Bull. Amer. Math. Soc., 19(2) (1988), 417-431, MR 0956596 (89k:57023), Zbl 0674.57008.

[21] M. Wada, Closed orbits of nonsingular Morse-Smale flows on $S^{3}$, J. Math. Soc. Japan, 41(3) (1989), 405-413, MR 0999505 (90g:58059), Zbl 0672.58042.

[22] R. Williams, One-dimensional nonwandering sets, Topology, 6 (1967), 473-487, MR 0217808 (36 \#897), Zbl 0159.53702.

Received July 1, 2003 and revised November 7, 2003. The first author was supported by NSF Grants \# DMS-0337713 and \# INT-0089631. The second author was supported in part by JSPS Research Fellowships for Young Scientists. 
Department of Mathematics

UNIVERSITY OF ILLINOIS

URBANA IL 61801

E-mail address: ghrist@math.uiuc.edu

Department of Mathematics

KYOTO UNIVERSITY

OIWAKE-CHO KitASHIRAKAWA SAKYO-KU KYOTO-SHI

КҮото 606-8502

JAPAN

E-mail address: kin@kusum.kyoto-u.ac.jp 